\title{
New frontiers in High-Value Care Education and Innovation: When Less is Not More
}

\author{
Pamela T Johnson, MD*; Amit Pahwa, MD; Daniel J Brotman, MD
}

Johns Hopkins University School of Medicine, Baltimore, Maryland.

$\mathrm{n}$ this issue of the Journal of Hospital Medicine ${ }^{\circledR}$, Drs. Arora and Moriates highlight an important deficiency in quality improvement efforts designed to reduce overuse of tests and treatments: the potential for trainees-and by extension, more seasoned clinicians - to rationalize minimizing under the guise of high-value care. ${ }^{1}$ This insightful perspective from the Co-Directors of Costs of Care should serve as a catalyst for further robust and effective care redesign efforts to optimize the use of all medical resources, including tests, treatments, procedures, consultations, emergency department (ED) visits, and hospital admissions. The formula to root out minimizers is not straightforward and requires an evaluation of wasteful practices in a nuanced and holistic manner that considers not only the frequency that the overused test (or treatment) is ordered but also the collateral impact of not ordering it. This principle has implications for measuring, paying for, and studying high-value care.

Overuse of tests and treatments increases costs and carries a risk of harm, from unnecessary use of creatine kinase-myocardial band (CK-MB) in suspected acute coronary syndrome ${ }^{2}$ to unwarranted administration of antibiotics for asymptomatic bacteriuria ${ }^{3}$ to over-administration of blood transfusions. ${ }^{4}$ However, decreasing the use of a commonly ordered test is not always clinically appropriate. To illustrate this point, we consider the evidence-based algorithm to deliver best practice in the workup of pulmonary embolism (PE) by Raja et al; which integrates pretest probability, PERC assessment, and appropriate use of D-dimer and pulmonary CT angiography (CTA). ${ }^{5}$ Avoiding D-dimer testing is appropriate in patients with very low pretest probability who pass a pulmonary embolism rule-out criteria (PERC) clinical assessment and is also appropriate in patients who have sufficiently high clinical probability for PE to justify CTA regardless of a D-dimer result. On the other hand, avoiding D-dimer testing by attributing a patient's symptoms to anxiety (as a minimizer might do) would increase patient risk, and could potentially increase cost if that patient ends up in intensive care after delayed diagnosis. Following diagnostic algorithms that include physician decision-making and evidence-based guidelines can prevent overuse and underuse, thereby maximizing efficiency and effectiveness. Engaging trainees in the development of

*Corresponding Author: Pamela T Johnson, MD; E-mail: PamelaJohnson@ jhmi.edu; Telephone: 410-336-0039; Twitter: @PamelaJohnsonMD.

Published online first April 8, 2019.

Received: December 18, 2019; Revised: January 27, 2019;

Accepted: February 4, 2019

@2019 Society of Hospital Medicine DOI 10.12788/jhm.3186 such algorithms and decision support tools will serve to ingrain these principles into their practice.

Arora and Moriates highlight the importance of caring for a patient along a continuum rather than simply optimizing practice with respect to a single management decision or an isolated care episode. This approach is fundamental to the quality of care we provide, the public trust our profession still commands, and the total cost of care (TCOC). The two largest contributors to debilitating patient healthcare debt are not overuse of tests and treatments, but ED visits and hospitalizations. ${ }^{6}$ Thus, high-value quality improvement needs to anticipate future healthcare needs, including those that may result from delayed or missed diagnoses. Furthermore, excessive focus on the minutiae of high-value care (fewer daily basic metabolic panels) can lead to change fatigue and divert attention from higher impact utilization. We endorse a holistic approach in which the lens is shifted from the test-and even from the encounter or episode of care-to the entire continuum of care so that we can safeguard against inappropriate minimization. This approach has started to gain traction with policymakers. For example, the state of Maryland has implemented a TCOC hospital payment model predicted to save $\$ 1$ billion by 2023 . $^{7}$ The TCOC model includes a Care Redesign Program whereby hospitals and nonhospital healthcare providers collaborate to improve the quality of care while reducing spending, and cost savings can be used for incentive payments to the nonhospital providers (gainsharing) while simultaneously monitoring quality measures to guard against rationing. ${ }^{7}$ In keeping with the authors' call to prioritize overall health, this new reimbursement model and others similar to it aim to incentivize the delivery of high-value care across a continuum.

Research is needed to guide best practice from this global perspective; as such, value improvement projects aimed at optimizing use of tests and treatments should include rigorous methodology, measures of downstream outcomes and costs, and balancing safety measures. ${ }^{8}$ For example, the ROMICAT II randomized trial evaluated two diagnostic approaches in emergency department patients with suspected acute coronary syndrome: early coronary computed tomography angiogram (CCTA) and standard ED evaluation. ${ }^{9}$ In addition to outcomes related to the ED visit itself, downstream testing and outcomes for 28 days after the episode were studied. In the acute setting, CCTA decreased time to diagnosis, reduced mean hospital length of stay by 7.6 hours, and resulted in $47 \%$ of patients being discharged within 8.6 hours as opposed to only $12 \%$ of the standard evaluation cohort. No cases of ACS were missed, and the CCTA cohort has slightly fewer cardiovascular adverse events $(P$ 
$=$.18). However, the CCTA patients received significantly more diagnostic and functional testing and higher radiation exposure than the standard evaluation cohort, and underwent modestly higher rates of coronary angiography and percutaneous coronary intervention. The TCOC over the 28-day period was similar at $\$ 4,289$ for CCTA versus $\$ 4,060$ for standard care $(P=.65){ }^{9}$

Reducing the TCOC is imperative to protect patients from the burden of healthcare debt, but concerns have been raised about the ethics of high-value care if decision-making is driven by cost considerations. ${ }^{10}$ A recent viewpoint proposed a framework where high-value care recommendations are categorized as obligatory (protecting patients from harm), permissible (call for shared decision-making), or suspect (entirely cost-driven). By reframing care redesign as thoughtful, responsible care delivery, we can better incentivize physicians to exercise professionalism and maintain medical practice as a public trust.

High-value champions have a great deal of work ahead to redesign care to improve health, reduce TCOC, and investigate outcomes of care redesign. We applaud Drs. Arora and Moriates for once again leading the charge in preparing medical students and residents to deliver higher-value healthcare by emphasizing that effective patient care is not measured by a single episode or clinical decision, but is defined through a lifelong partnership between the patient and the healthcare system. As the country moves toward improved holistic models of care and financing, physician leadership in care redesign is essential to ensure that quality, safety, and patient well-being are not sacrificed at the altar of cost savings.

Disclosures: Dr. Johnson is a Consultant and Advisory Board Member at Oliver Wyman, receives salary support from an AHRQ grant, and has pending poten- tial royalties from licensure of evidence-based appropriate use guidelines/criteria to AgilMD (Agile is a clinical decision support company). The other authors have no relevant disclosures. Dr. Johnson and Dr. Pahwa are Co-directors, High Value Practice Academic Alliance, www.hvpaa.org

\section{References}

1. Arora $V$, Moriates $C$. Tackling the minimizers behind high value care. J Hos Med. 2019: 14(5):318-319. doi: 10.12788/jhm.3104

2. Alvin MD, Jaffe AS, Ziegelstein RC, Trost JC. Eliminating creatine kinase-myocardial band testing in suspected acute coronary syndrome: a value-based quality improvement. JAMA Intern Med. 2017;177(10):1508-1512. doi: 10.1001/jamainternmed.2017.3597.

3. Daniel M, Keller S, Mozafarihashjin M, Pahwa A, Soong C. An implementation guide to reducing overtreatment of asymptomatic bacteriuria. JAMA Intern Med. 018;178(2):271-276. doi: 10.1001/jamainternmed.2017.7290.

4. Sadana D, Pratzer A, Scher LJ, et al. Promoting high-value practice by reducing unnecessary transfusions with a patient blood management program. JAMA Intern Med. 2018;178(1):116-122. doi: 10.1001/jamainternmed.2017.6369.

5. Raja AS, Greenberg JO, Qaseem A, et al. Evaluation of patients with suspected acute pulmonary embolism: Best practice advice from the Clinical Guidelines Committee of the American College of Physicians. Ann Intern Med. 2015;163(9):701-711. doi: 10.7326/M14-1772

6. The Burden of Medical Debt: Results from the Kaiser Family Foundation/ New York Times Medical Bills Survey. https://www.kff.org/health-costs/report/the-burden-of-medical-debt-results-from-the-kaiser-family-foundationnew-york-times-medical-bills-survey/. Accessed December 2, 2018.

7. Maryland Total Cost of Care Model. https://innovation.cms.gov/initiatives/ md-tccm/. Accessed December 2, 2018

8. Grady D, Redberg RF, O'Malley PG. Quality improvement for quality improvement studies. JAMA Intern Med. 2018;178(2):187. doi: 10.1001/jamainternmed.2017.6875.

9. Hoffmann U, Truong QA, Schoenfeld DA, et al. Coronary CT angiography versus standard evaluation in acute chest pain. N Engl J Med. 2012;367:299308. doi: 10.1056/NEJMoa1201161.

10. DeCamp M, Tilburt JC. Ethics and high-value care. J Med Ethics. 2017;43(5):307-309. doi: 10.1136/medethics-2016-103880. 